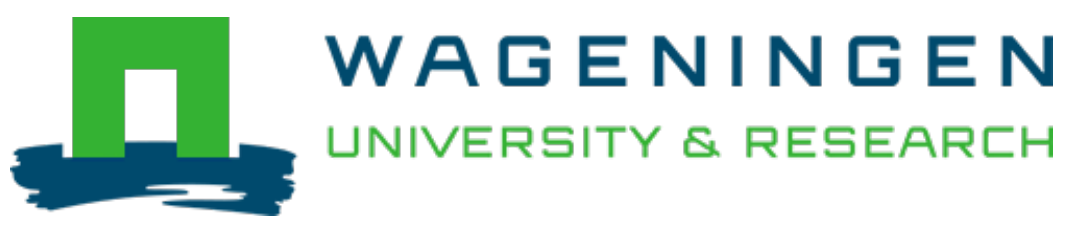

\title{
A biaryl-linked tripeptide from Planomonospora reveals a widespread class of minimal RiPP gene clusters
}

\author{
Cell Chemical Biology \\ Zdouc, Mitja M.; Alanjary, M.M.; Zarazúa, Guadelupe S.; Maffioli, Sonia I.; Crüsemann, M. et al \\ https://doi.org/10.1016/j.chembiol.2020.11.009
}

This article is made publicly available in the institutional repository of Wageningen University and Research, under the terms of article $25 \mathrm{fa}$ of the Dutch Copyright Act, also known as the Amendment Taverne. This has been done with explicit consent by the author.

Article 25 fa states that the author of a short scientific work funded either wholly or partially by Dutch public funds is entitled to make that work publicly available for no consideration following a reasonable period of time after the work was first published, provided that clear reference is made to the source of the first publication of the work.

This publication is distributed under The Association of Universities in the Netherlands (VSNU) 'Article $25 \mathrm{fa}$ implementation' project. In this project research outputs of researchers employed by Dutch Universities that comply with the legal requirements of Article $25 \mathrm{fa}$ of the Dutch Copyright Act are distributed online and free of cost or other barriers in institutional repositories. Research outputs are distributed six months after their first online publication in the original published version and with proper attribution to the source of the original publication.

You are permitted to download and use the publication for personal purposes. All rights remain with the author(s) and / or copyright owner(s) of this work. Any use of the publication or parts of it other than authorised under article $25 \mathrm{fa}$ of the Dutch Copyright act is prohibited. Wageningen University \& Research and the author(s) of this publication shall not be held responsible or liable for any damages resulting from your (re)use of this publication.

For questions regarding the public availability of this article please contact openscience.library@,wur.nl 


\section{Cell Chemical Biology}

\section{A biaryl-linked tripeptide from Planomonospora reveals a widespread class of minimal RiPP gene clusters}

\section{Graphical Abstract}
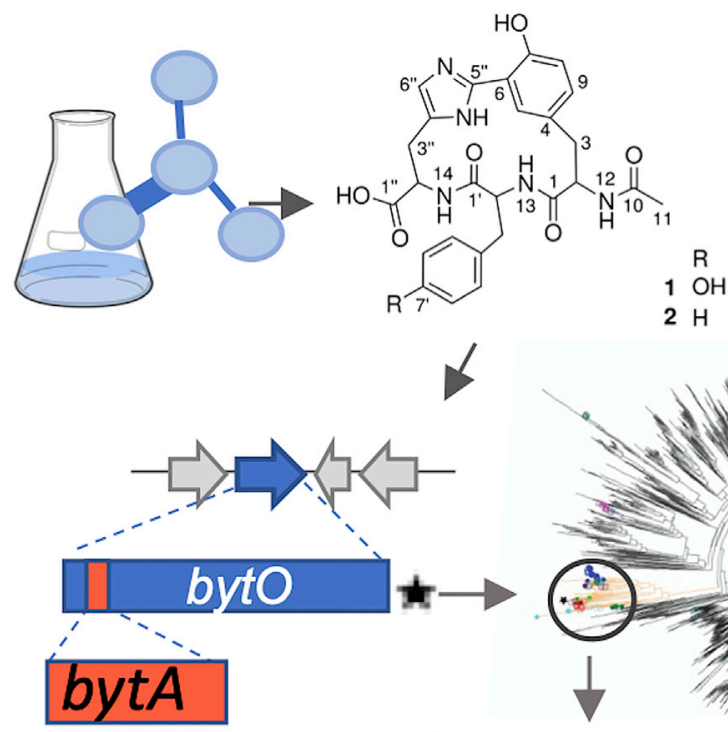

MRYYH

\section{$\mathrm{M} \leq \mathrm{Y}$ wH}

$2 \mathrm{H}$
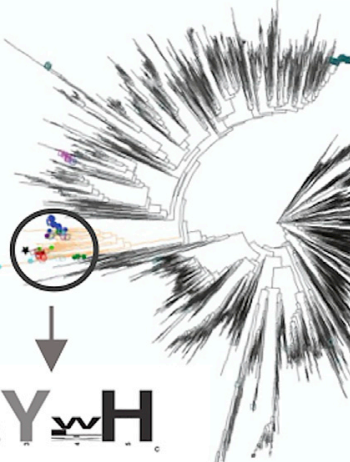

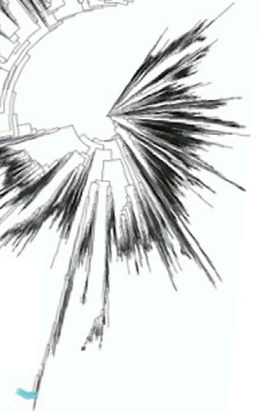

Highlights

- N-Acetylated tripeptides with an unusual Tyr-His biaryl bridging

- Ribosomally synthetized from a pentapeptide precursor and modified by a cytochrome P450

- Encoded by the 18-bp bycA gene, the smallest coding gene ever reported

- Widespread distribution of related two-gene clusters in actinobacterial genomes

\section{Authors}

Mitja M. Zdouc, Mohammad M. Alanjary, Guadalupe S. Zarazúa, ..., Marnix H. Medema, Stefano Donadio, Margherita Sosio

\section{Correspondence}

mzdouc@naicons.com (M.M.Z.), msosio@naicons.com (M.S.)

\section{In Brief}

Zdouc et al. describe the discovery of two crosslinked, cyclic tripeptides with unusual Tyr-His biaryl bridging. Their formation requires a minimal biosynthetic gene cluster consisting of a gene encoding a pentapeptide precursor and a gene encoding cytochrome P450 monooxygenase. Phylogenomic analysis revealed widespread distribution of related two-gene clusters in actinobacterial genomes. 


\title{
A biaryl-linked tripeptide from Planomonospora reveals a widespread class of minimal RiPP gene clusters
}

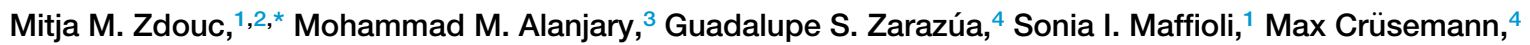 \\ Marnix H. Medema, ${ }^{3}$ Stefano Donadio, ${ }^{1}$ and Margherita Sosio ${ }^{1,5, *}$ \\ ${ }^{1}$ Naicons Srl., Viale Ortles 22/4, 20139 Milano, Italy \\ 2Swammerdam Institute for Life Sciences, University of Amsterdam, Science Park 904, Amsterdam, XH 1098, the Netherlands \\ ${ }^{3}$ Bioinformatics Group, Wageningen University, Droevendaalsesteeg 1, Wageningen, PB 6708, the Netherlands \\ ${ }^{4}$ Institut für Pharmazeutische Biologie, Rheinische Friedrich-Wilhelms-Universität, Nußallee 6, Bonn 53115, Germany \\ ${ }^{5}$ Lead Contact \\ *Correspondence: mzdouc@naicons.com (M.M.Z.), msosio@naicons.com (M.S.) \\ https://doi.org/10.1016/j.chembiol.2020.11.009
}

\section{SUMMARY}

Microbial natural products impress by their bioactivity, structural diversity, and ingenious biosynthesis. While screening the less exploited actinobacterial genus Planomonospora, two cyclopeptides were discovered, featuring an unusual Tyr-His biaryl bridging across a tripeptide scaffold, with the sequences $\mathrm{N}$-acetyl-TyrTyr-His and N-acetyl-Tyr-Phe-His. Planomonospora genomes pointed toward a ribosomal synthesis of the cyclopeptide from a pentapeptide precursor encoded by 18-bp bytA, to our knowledge the smallest coding gene ever reported. Closely linked to byt $A$ is bytO, encoding a cytochrome $\mathrm{P} 450$ monooxygenase likely responsible for biaryl installment. In Streptomyces, the bytAO segment was sufficient to direct production of the crosslinked $\mathrm{N}$-acetylated Tyr-Tyr-His tripeptide. Bioinformatic analysis of related cytochrome P450 monooxygenases indicated that they constitute a widespread family of enzymes, and the corresponding genes are closely linked to 5-amino acid coding sequences in approximately 200 (actino)bacterial genomes, all with potential for biaryl linkage between amino acids 1 and 3 . We propose the named biarylitides this family of RiPPs.

\section{INTRODUCTION}

Microbial natural products impress not only for their potent bioactivity, but also for their chemical diversity and originality. Structurally novel compounds have a high chance of interacting with novel targets or with mechanisms of action different from known compounds (Yera et al., 2011; Gfeller et al., 2013; Chen et al., 2020). In recent years, ribosomally synthesized and post-translationally modified peptides (RiPPs) have drawn attention due to their intricate, three-dimensional (3D) structures and wide distribution in bacteria. RiPP biosynthetic gene clusters (BGCs) are compact, encompassing only a precursor peptide, separated in an $\mathrm{N}$-terminal leader and a $\mathrm{C}$-terminal core peptide. After ribosomal translation, the precursor peptide is modified by dedicated enzymes, with consecutive cleavage of the leader peptide and release of the mature core peptide (Arnison, et al., 2013; Funk and van der Donk, 2017). RiPPs are grouped into families on the basis of their structural features and post-translational modifications (Funk and van der Donk, 2017). Several bioinformatic tools have been developed for the automatic recognition of RiPP BGCs in microbial genomes, which are usually based on recognizing elements, such as leader peptides or diagnostic RiPP-processing enzymes (Russell and Truman, 2020).
In this study, we identified two crosslinked, N-acetylated tripeptides featuring an unusual Tyr1-His3 biaryl bridging. Genome analysis of the producing strains led to the identification of the shortest RiPP BGC so far reported, encoding just a pentapeptide precursor and a closely linked cytochrome P450 monooxygenase. Phylogenomic analysis of the latter sequence revealed that cytochrome P450 monooxygenase genes closely linked to a 5-amino acid coding sequence represent a widespread family of minimal RiPP BGCs present especially in actinobacterial genomes.

\section{RESULTS AND DISCUSSION}

Identification of crosslinked N-Acetylated Tyr-Tyr-His In the search for microbial secondary metabolites, we investigated 72 strains belonging to the poorly characterized actinomycete genus Planomonospora (Monciardini et al., 2014) by extensive liquid chromatography coupled to high-resolution ion electrospray ionization-tandem mass spectrometry-based metabolome mining of extracts (Zdouc et al., 2020). Molecular networking analysis revealed a group of related features (see Figure S1) observed in samples from four phylogenetically related strains, with $16 \mathrm{~S}$ rRNA gene sequences having $99.5 \%-$ 99.6\% identity with Planomonospora algeriensis PM3 
A

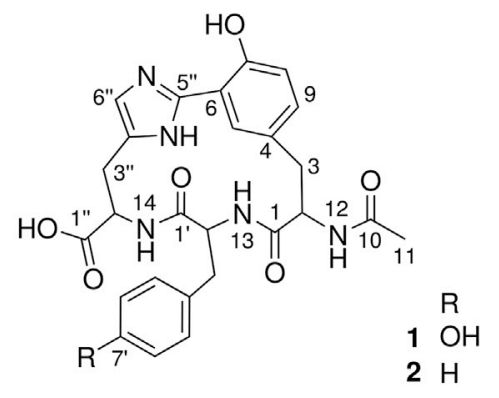

B

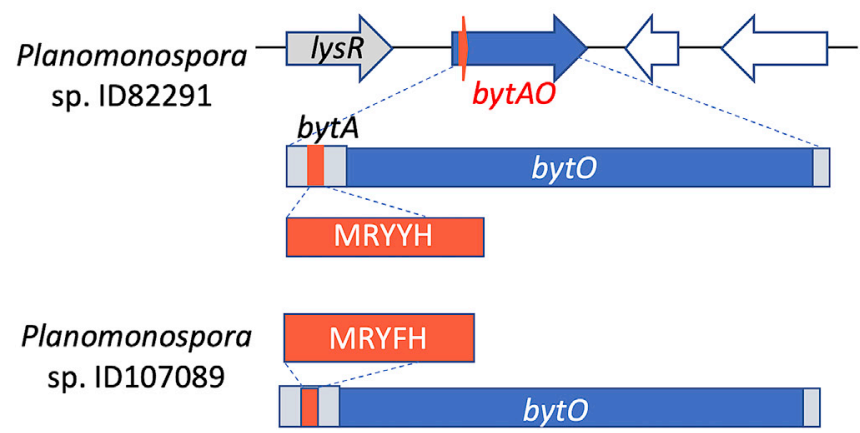

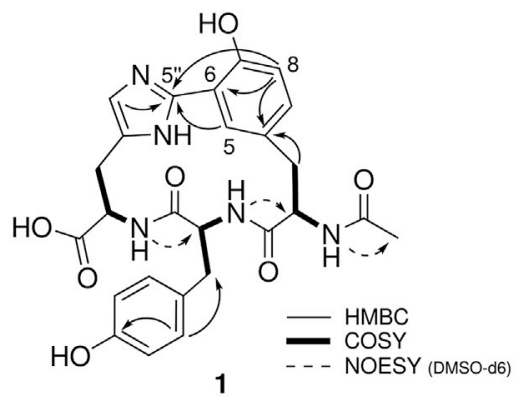

C

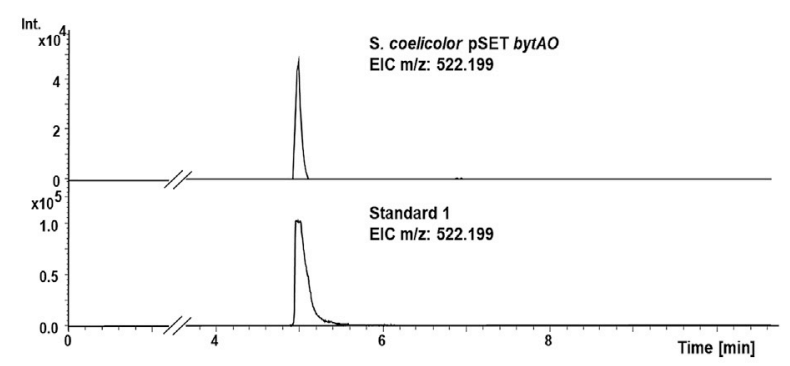

Figure 1. Chemical structures of 1 and 2, the bytAO region for 1 and 2 biosynthesis, and its heterologous expression (A) Structures of 1 and 2 with key 2D NMR correlations for 1 (right side); (B) bytA encodes the 5 -amino acid precursor peptide, while bytO encodes the cytochrome P450 monooxygenase PLM4_2056. A lysR family transcriptional regulator is in the vicinity of bytAO and the two empty arrows represent genes outside the 1 biosynthetic cluster. The corresponding segment from the genome of Planomonospora sp. ID107089 is shown at the bottom. Note amino acid difference in BytA; (C) extracted ion chromatograms (EIC) from heterologous expression of bytAO in Streptomyces coelicolor M1152 (top) and an authentic standard of 1 (bottom). See also Figures $\mathrm{S} 1$ and $\mathrm{S} 4$ and Table $\mathrm{S} 1$.

(Zdouc et al., 2020). One strain, Planomonospora sp. ID82291, produced a compound (1) with an $\mathrm{m} / \mathrm{z} 522.198[\mathrm{M}+\mathrm{H}]^{+}$(calculated molecular formula $\mathrm{C}_{26} \mathrm{H}_{28} \mathrm{~N}_{5} \mathrm{O}_{7}$ ), while the other three strains produced a compound (2) with an $\mathrm{m} / \mathrm{z} 506.203[\mathrm{M}+\mathrm{H}]^{+}$ (calculated molecular formula $\mathrm{C}_{26} \mathrm{H}_{28} \mathrm{~N}_{5} \mathrm{O}_{6}$ ). Additional variants were found only in extracts from strain ID46116 (Figure S1) and were not further investigated. $\mathbf{1}$ and $\mathbf{2}$ showed UV absorption maxima at 270 and $315 \mathrm{~nm}$, the latter indicating an unusual chromophore.

Planomonospora sp. ID82291 was selected for further investigation. From a 15-L culture, $5 \mathrm{mg}$ pure 1 were isolated. Extensive 1D and 2D NMR analyses indicated an acetylated short peptide. A first set of experiments, performed in DMSO-d6, allowed the observation of three aromatic spin systems containing $\mathrm{CH} \alpha$ and $\mathrm{CH}_{2} \beta$ consistent with two tyrosine and one histidine residues. The correlations observed in a NOESY experiment suggested the sequence $\mathrm{N}$-acetyl-Tyr-Tyr-His, but with an additional unsaturation, which could not be assigned due to overlapping aromatic signals. A second set of analyses in $\mathrm{CD}_{3} \mathrm{OD}$ allowed the complete assignment of the non-exchangeable protons of 1. TOCSY and HSQC experiments showed that the histidine residue lacked its characteristic aromatic proton at position $5^{\prime \prime}$ but had a quaternary carbon at $142 \mathrm{ppm}$. In an HMBC experiment, a diagnostic cross-correlation was observed between both $\mathrm{CH}-5$ and $\mathrm{CH}-8$ and $\mathrm{C}^{\prime \prime}$, consistent with a carbon-carbon bond between $\mathrm{C} 6$ and $\mathrm{C5}^{\prime \prime}$ (Figure 1A). The proton and carbon assignments and the 1D and 2D spectra are reported in Figures S2 and S3 and Table S1.

The existence of the crosslink was confirmed after hydrolysis of 1 , which led to the release of a compound with $m / z 335[\mathrm{M}+$ $\mathrm{H}]^{+}$, corresponding to the expected carbon-carbon-linked tyrosine and histidine, and a compound with $m / z 480[\mathrm{M}+\mathrm{H}]^{+}$, consistent with the loss of the acetyl group from the intact tripeptide. The $\mathrm{m} / \mathrm{z} 335[\mathrm{M}+\mathrm{H}]^{+}$compound retained the UV maximum at $315 \mathrm{~nm}$, as expected for the biaryl chromophore.

Compound 2, produced by the other three Planomonospora isolates, was deduced to be $\mathrm{N}$-acetyl-Tyr-Phe-His, based on the similarity of the tandem mass fragmentation of $\mathbf{1}$ and $\mathbf{2}$, with a neutral loss corresponding to phenylalanine $(-147.07$ $\mathrm{Da}$ ) being the only difference between the fragmentation spectra (see Table S2).

We could only identify one literature precedent for natural products with a tyrosine-histidine biaryl bridge: aciculitins, cyclic nonapeptides decorated with glycolipids from the marine sponge Aciculites orientalis (Bewley et al., 1996). They contain a carboncarbon bond between tyrosine and histidine but with a connectivity different than in 1. Interestingly, aciculitins, which lack additional aromatic residues, show UV absorption maxima at 270 and $310 \mathrm{~nm}$, matching those of 1 and 2 (Bewley et al., 1996).

A few metabolites have been described that consist of a crosslinked (N-acetylated) tripeptide: $\mathrm{K}-13$ (3) from Micromonospora halophytica ssp. exilisia with a Tyr-Tyr-Tyr sequence and an ether 
<smiles>CC(=O)NC(Cc1ccc(Oc2cccc(CC(NC(=O)C(Cc3ccc(O)cc3)Cc3ccc(O)cc3)C(=O)O)c2)cc1)C(=O)O</smiles><smiles>NC(=O)CC(NC(=O)[C@H](N)Cc1ccc(O)c(Oc2ccc(C[C@@H](NC(=O)O)C(=O)O)cc2)c1)C(=O)O</smiles>

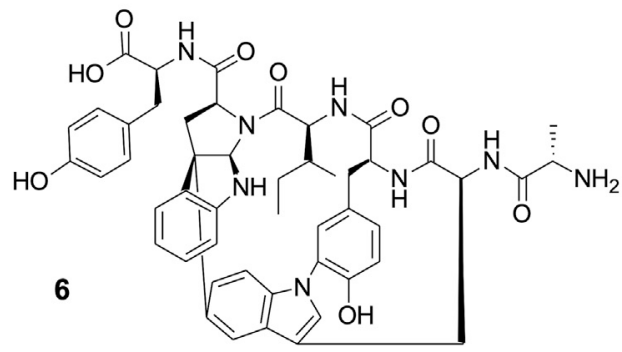

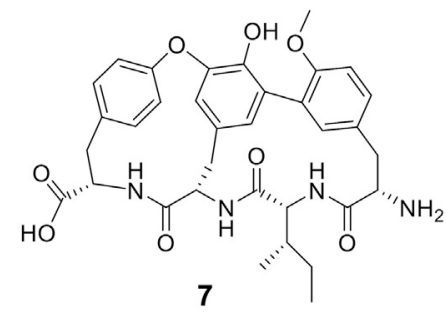

Figure 2. Structures of relevant metabolites mentioned in the text

K-13 (3), OF-4949 (4), pseudosporamide (5), tryptorubin (6), and cittilin A (7). Note that four variants of OF-4949 have been reported, with either $\mathrm{H}$ or $\mathrm{Me}$ on Tyr1-OH and $\mathrm{H}$ or $\mathrm{OH}$ on the Asn $\beta$ carbon, but only one is shown for simplicity.

(see below), is similar to the recently reported BGC for tryptorubin (6), a hexapeptide containing carbon-carbon and carbon-nitrogen bonds between aromatic amino acid residues (Wyche et al., 2017; Reisberg et al., 2020). Also for 6, the BGC encodes just one cytochrome P450 enzyme and a precursor peptide, the latter, however, containing a 22amino acid leader peptide. Similarly, the recently reported $B G C$ for cittilin $A(7)$, a crosslinked YIYY tetrapeptide from Myxococcus xanthus, encodes a 27-amino acid precursor peptide, an adjacent cyto-

bond between the oxygen of Tyr-3 and C6 of Tyr-1 (Kase et al., 1987; Yasuzawa et al., 1987); OF-4949 (4) from Penicillinum rugulosum OF4949, which is identical to $\mathbf{3}$ except for asparagine in place of the central tyrosine and lack of the N-terminal acetyl group (Sano et al., 1986); and pseudosporamide (5) from Pseudosporangium sp. RD062863, reported while writing this article, with a Tyr-Pro-Trp sequence and a carbon-carbon bond between C6 of tyrosine and C-9 of tryptophan (Saito et al., 2020). Of note, compounds $\mathbf{3}, \mathbf{4}$, and $\mathbf{5}$ lack an absorption maximum at $315 \mathrm{~nm}$.

It should be noted that compounds $\mathbf{3}$ to $\mathbf{5}$ have been shown to consist of L-amino acids only, consistent with a ribosomal synthesis. Among the 1-related metabolites, $\mathbf{3}$ and $\mathbf{4}$ have been reported to inhibit the proteases angiotensin-converting enzyme (ACE) and aminopeptidase B, respectively, with apparent strict specificity, since 4 is not an ACE inhibitor. We were unable to observe inhibition of different proteases by 1 (data not shown), suggesting that the amino acid sequence and/or ring size can strongly influence activity. 5 was reported to have weak cytotoxic activity (Saito et al., 2020).

When the strain was grown in $\mathrm{D}_{2} \mathrm{O}$-supplemented medium, 1 was found to be extensively labeled with deuterium (Figure S4), indicating that its formation requires de novo amino acid synthesis.

We are unaware of any biosynthetic studies on $\mathbf{3 , 4}$, or $\mathbf{5}$. To get insights into 1 biosynthesis, we analyzed the 7.58-Mbp genome of Planomonospora sp. ID82291 (Zdouc et al., 2020) for the presence of antiSMASH-predicted NRPS or RiPP BGCs (Blin, et al., 2019), likely to specify a Tyr-Tyr-His peptide. However, this search proved unsuccessful, suggesting that 1 was formed by a BGC not recognized by the antiSMASH search tools. Searching the six-frame translation of the ID82291 genome led to a single plausible candidate: a short open reading frame encoding the pentapeptide MRYYH, preceded by a ribosomal binding site and followed by PLM4_2056, encoding a cytochrome P450 monooxygenase (see Figure 1B). The proposed BGC for 1, encoding the two genes designated byt $A$ and bytO chrome P450 enzyme, a methyltransferase and a distant endopeptidase for cleavage (Hug et al., 2020). Cytochrome P450 monooxygenases are known to install crosslinks between aromatic residues of peptides, such as, e.g., in the NRPS-generated glycopeptides (Greule et al., 2018). These precedents made the BGC depicted in Figure 1B as the likely candidate for $\mathbf{1}$ biosynthesis. Apart from a possible regulator, the five genes upstream and downstream bytAO do not partake in $\mathbf{1}$ biosynthesis.

A similar two-gene organization was found in the genome of Planomonospora sp. ID107089, one of the producers of 2 (Figures $1 \mathrm{~B}$ and $\mathrm{S} 1$ ). Of note, the two bytAO regions are almost identical: there is only one nucleotide $(A-T)$ difference between the byt $A$ genes, responsible for the $\mathrm{Y}-\mathrm{F}$ difference in the peptides; both genes are preceded by equally spaced, identical ribosomal binding sites; the bytO sequences share $97 \%$ identity, while the bytAO intergenic regions (120 bp) are 95\% identical.

\section{Heterologous expression of bytAO}

To prove that production of $\mathbf{1}$ is indeed governed by bytAO, we cloned a 1,303-bp sequence containing byt $A$ and bytO into pSET152 and conjugated the plasmid into the expression host Streptomyces coelicolor M1152. Cultivation of S. coelicolor pSET bytAO, followed by HP20 extraction and high-performance liquid chromatography-tandem mass spectrometry analysis, resulted in the detection of a peak with an $\mathrm{m} / \mathrm{z} 522.199$, having the same UV spectrum, retention time, and tandem mass spectrometry spectrum as $\mathbf{1}$ (Figures $1 \mathrm{C}$ and $\mathrm{S} 4$ ). This unambiguously confirms the heterologous production of mature 1 by the minimal gene cluster bytAO, making it the shortest RiPP BGC so far reported. To our knowledge, the shortest reported peptide-encoding gene is the 24-bp mccA, which encodes the precursor peptide of microcin C7 in Escherichia coli, a RiPP also devoid of leader peptide (González-Pastor et al., 1994; Guijarro et al., 1995). With just 18 bp (including the stop codon), byt $A$ is two codons smaller than $m c c A$ and thus the smallest peptide-coding gene ever reported across the Tree of Life. Since heterologous expression of bytAO 


\section{CellPress}

\section{Cell Chemical Biology Brief Communication}

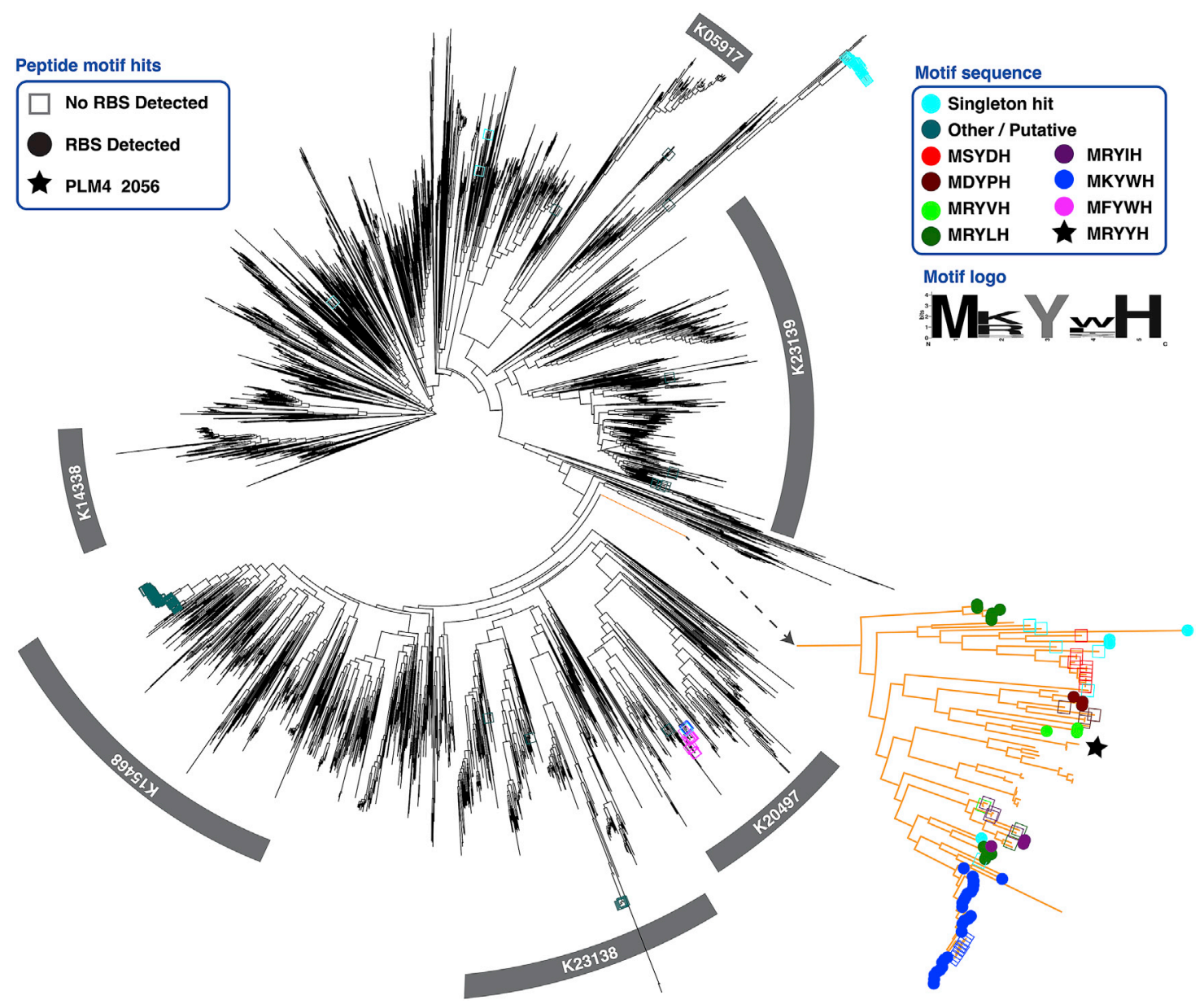

Figure 3. Overview phylogeny of cytochrome P450s from a variety of bacterial genera used in this study

KEGG orthology IDs in gray boxes highlight selected functional groupings (K20497: methyl-branched lipid omega-hydroxylase; K23138: family CYP109; K23139: family CYP110; K05917: steroid biosynthesis; K15468: polyketide biosynthesis; K14338: fatty acid degradation). Positive matches for pentapeptide motif hits are indicated as circles, where filled ones visualize a fully validated Shine-Dalgarno (SD) ribosomal binding site (RBS), while for empty ones an SD sequence was not detected. Putative singleton hits are marked in light or dark cyan if the same motif is not seen in other P450s, with <95\% amino acid identity, or if the hit overlaps with the P450 coding region, respectively. Identical motifs correspond to the same color shown in the legend. P450s likely associated with biarylitides are highlighted in orange. This clade also contains the bytAO sequence marked with a filled star. The related bytAO sequence from strain ID107089 has not been included for simplicity. Consensus sequences of all motifs with validated RBS sites are illustrated with the sequence logo at the bottom right. More annotations and interactive tree can be accessed at: https://itol.embl.de/tree/21312710611256691590408100.

See also Figure S4.

in $S$. coelicolor resulted in the mature product, we infer that 1 biosynthesis recruits one or more generic enzymes, involved in trimming the additional amino acid(s) at the $\mathrm{N}$ terminus and in $\mathrm{N}-$ acetylation. Of note, the existence of cluster-encoded proteases in RiPP BGCs from Actinobacteria seems to be the exception (Chen et al., 2019). As an additional observation, the $\mathrm{N}$-acetylated compounds depicted in Figure 2 are all produced by Actinobacteria, while those with a free $\mathrm{N}$ terminus were observed in fungi or myxobacteria.

Biarylitides: a widespread family of RiPPs

We reasoned that the cytochrome P450 monooxygenase PLM4_2056 would be selective in installing a biaryl crosslink on short peptides, and that it could belong to a distinct phylogenetic branch of this enzyme family. If the cognate pentapeptide-encoding gene was closely linked to the cytochrome monooxygenase gene, then one could easily identify additional BGCs related to those depicted in Figure 1B. We thus conducted a search for analogous small CDSs (coding DNA sequences) in the flanking regions of genes related to PLM4_2056. Assuming structural conservation of the carbon-carbon bond, we restricted our search to pentapeptides with tyrosine at position 3 and tyrosine/histidine at position 5. From approximately 3,300 PLM_2056-related sequences, CDSs encoding the specified peptides were identified in approximately 200 genomes, the majority from Actinobacteria. To increase our confidence in the hits, we filtered the detected CDS for a Shine-Dalgarno motif 6-8 bp upstream of the peptide sequence, as indicated in the phylogenetic trees shown in Figures 3 and 4. Interestingly, such a ribosomal binding site was only detected in sequences from Actinobacteria, where it occurred in the vicinity of approximately half of the detected peptides (indicated by filled circles 


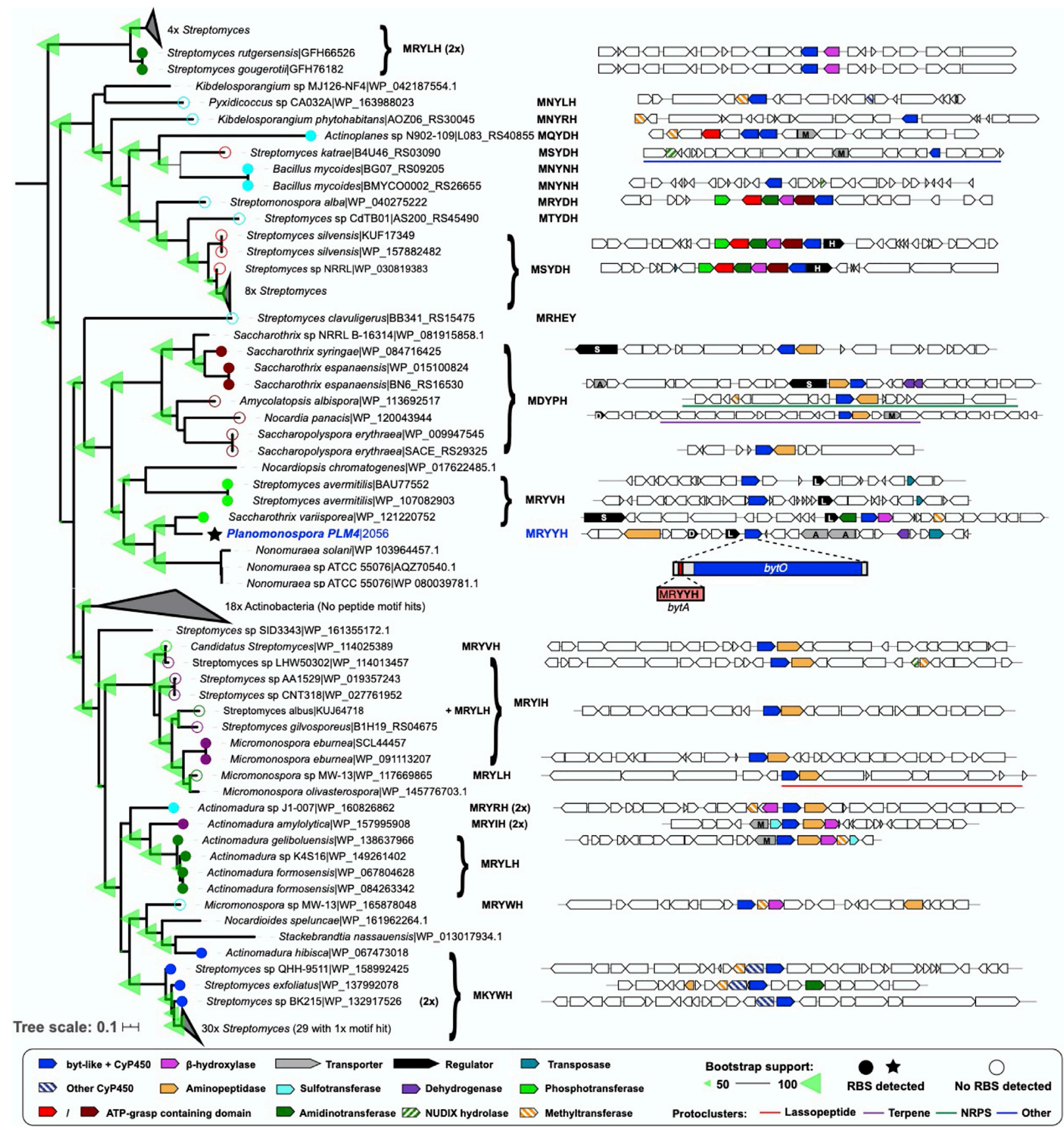

Figure 4. Summary phylogeny of cytochrome P450s, likely associated with biarylitide biosynthesis

Identical pentapeptide motif hits are shown as colored circles corresponding to the motif printed to the right of the tree. Filled and unfilled circles indicate those with and without a detected Shine-Dalgarno RBS, respectively. Selected regions of byt-like clusters are shown to the right of motifs, illustrating a variety of contexts and shared coding sequences potentially linked to the minimal P450 cluster (highlighted in blue). Expanded clades and detailed annotations are accessible at: https://itol.embl.de/tree/2131271061176991591098016 (Letunic and Bork, 2019).

in Figures 3 and 4). Furthermore, this clade was seen to have the only example of replicate motifs (denoted " $2 x$ " in Figure 4). To get insight into the amino acid distribution, detected peptides were aligned using the program MUSCLE. Peptides with a pre- ceding ribosomal binding site showed less variation, with a strong preference for a basic amino acid at position 2, in comparison with the global alignment (see Figures 3 and S4). Intriguingly, the diversity of genomic loci in which PLM_2056 homologs 


\section{CellPress}

were found together with bytA-like motifs, suggests that these BGCs encode the production of a much wider diversity of molecules with distinct chemical modifications, as suggested by the co-localization of methyltransferases, sulfotransferases, ATPgrasp enzymes, and other tailoring enzymes in these loci (Figure 4). Together, this represents a treasure trove for further discovery efforts in this unexplored family.

As a side observation, sequence identity between BytO and actinobacterial P450 monooxygenases from the biarylitides clade ranges between $40 \%$ and $50 \%$. In contrast, BytO shows $20 \%-25 \%$ identity with P450 monooxygenases involved in glycopeptide crosslinks.

We propose the name biarylitides for this family of $\mathrm{N}$-acetylated tripeptides, carrying a crosslink between the aromatic side chains of amino acids 1 and 3 . Accordingly, compounds $\mathbf{1}$, 2, K-13, and pseudosporamide would become biarylitide $Y Y H$, YFH, YYY, and YPW, respectively. Since the first comprehensive literature overview of just a few years ago (Arnison et al., 2013), several new RiPP families have been uncovered, mostly through searching precursor peptide-related features in genome sequences (Kloosterman et al., 2020; Montalbán-López et al., 2020). RiPPs such as the biarylitides can, however, not be detected by current bioinformatic search strategies because of the extremely small size of the bytA gene. This highlights the importance of metabolomics approaches to unveil novel chemistry and unexpected BGCs. As shown here and recently described in Russell and Truman (2020), with hindsight from genomic data, these minimal RiPPs can be identified via cytochrome P450 homology and screening for the short peptide sequences in many bacterial genomes. While occurring mostly in Actinobacteria, one such BGC was also detected in a Pyxidicoccus (myxobacteria) strain. While we restricted our searches to a pentapeptide $\mathrm{MxYxY/H}$-encoding gene within $\pm 500 \mathrm{bp}$ from a cytochrome P450 monooxygenase gene, the similar architecture of BGCs for tryptorubin and cittilin A suggests that many further variations in short, crosslinked peptides exists in microbial genomes. Additional search strategies are thus likely to further expand these RiPP families.

While the byt BGC is extremely small, it poses nonetheless some biochemical challenges for the specificity of the biaryl crosslink. The basic amino acid, often found at position -1 , might act as a signal for processing. Further studies will be needed to understand the timing and sequence requirements for installing a crosslink and trimming the additional amino acid(s) at the $\mathrm{N}$ terminus. Since the biaryl crosslink imparts proteolytic stability, understanding its specificity might have important applications in stabilizing peptides for different applications. Our understanding of the biological activities of biarylitides is extremely limited. The different protease inhibitory activities seen with $\mathbf{1}, \mathbf{3}$, and $\mathbf{4}$ suggest that the amino acid sequence and/or ring size can strongly influence bioactivity. Thus, their biological properties and their role in the producing organisms await discovery.

\section{SIGNIFICANCE}

We have identified crosslinked, cyclic tripeptides produced by different strains of Planomonospora and established that its formation in a heterologous host requires a minimal biosynthetic gene cluster consisting of just two genes: one encoding a pentapeptide, representing the smallest peptide-coding gene ever reported, and the other encoding a cytochrome P450 monooxygenase. Phylogenomic analysis of related cytochrome P450 sequences revealed that this class of minimal RiPP gene clusters is widespread in actinobacterial genomes.

\section{STAR $\star$ METHODS}

Detailed methods are provided in the online version of this paper and include the following:

- KEY RESOURCES TABLE

- RESOURCE AVAILABILITY

Lead contact

O Materials availability

- Data and code availability

- EXPERIMENTAL MODEL AND SUBJECT DETAILS

O Bacterial strains and growth conditions

$\circ$ Cultivation in $\mathrm{D}_{2} \mathrm{O}$

- METHOD DETAILS

Isolation and purification of 1

Analytical procedures

Hydrolysis of 1

O Biarylitide YYH (1)

Cloning and heterologous Expression of bytAO

O Protease activity assay

O Phylogenetic analyses

O Peptide motif search

- QUANTIFICATION AND STATISTICAL ANALYSIS

\section{SUPPLEMENTAL INFORMATION}

Supplemental Information can be found online at https://doi.org/10.1016/j. chembiol.2020.11.009.

\section{ACKNOWLEDGMENT}

The authors thank Stefan Kehraus for measuring optical rotation of 1. This work has received funding from the European Union's Horizon 2020 research and innovation program under grant agreement no. 721484 (Train2Target). G.S.Z. acknowledges the DAAD for a PhD scholarship. M.C. received funding from the German Research Foundation (DFG) grant no. FOR2372. M.M.A. and M.H.M. were supported by an ERA NET CoBiotech (Bestbiosurf) grant through the Netherlands Organization for Scientific Research (NWO) (053.80.739).

\section{AUTHOR CONTRIBUTIONS}

M.M.Z., S.D., and M.S. designed the work, identified metabolites, analyzed the data, and wrote the paper. M.M.A. and M.H.M. performed bioinformatics analyses. G.S.Z. and M.C. performed heterologous expression and analyzed the data. S.I.M. identified metabolites and analyzed the data. All authors contributed to the critical discussion of the manuscript, and read and approved the final manuscript.

\section{DECLARATION OF INTERESTS}

M.M.Z., M.S., S.I.M., and S.D. have been and/or are employees and/or shareholders of Naicons Srl. M.H.M. is a co-founder of Design Pharmaceuticals and a member of the scientific advisory board of Hexagon Bio. The other authors declare that no competing interests exist. 


\section{Cell Chemical Biology Brief Communication}

Received: September 11, 2020

Revised: October 23, 2020

Accepted: November 20, 2020

Published: December 14, 2020

\section{REFERENCES}

Arnison, P.G., Bibb, M.J., Bierbaum, G., Bowers, A.A., Bugni, T.S., Bulaj, G., Camarero, J.A., Campopiano, D.J., Challis, G.L., Clardy, J., et al. (2013). Ribosomally synthesized and post-translationally modified peptide natural products: overview and recommendations for a universal nomenclature. Nat. Prod. Rep. 30, 108-160.

Bewley, C.A., He, H., Williams, D.H., and Faulkner, D.J. (1996). Aciculitins A-C: cytotoxic and antifungal cyclic peptides from the lithistid sponge Aciculites orientalis. J. Am. Chem. Soc. 118, 4314-4321.

Bierman, M.I., Stein, K.L., and Snyder, J.V. (1992). Plasmid cloning vectors for the conjugal transfer of DNA from Escherichia coli to Streptomyces spp. Gene 116, 43-49

Blin, K., Shaw, S., Steinke, K., Villebro, R., Ziemert, N., Lee, S.Y., Medema, M.H., and Weber, T. (2019). antiSMASH 5.0: updates to the secondary metabolite genome mining pipeline. Nucleic Acids Res. 2, W81-W87, https://doi.org/ 10.1093/nar/gkz310.

Chen, Y., Mathai, N., and Kirchmair, J. (2020). Scope of 3D shape-based approaches in predicting the macromolecular targets of structurally complex small molecules including natural products and macrocyclic ligands. J. Chem. Inf. Model. 60, 2858-2875, https://doi.org/10.1021/acs.jcim. 0c00161.

Chen, S., Xu, B., Chen, E., Wang, J., Lu, J., Donadio, S., Ge, H., and Wang, H. (2019). Zn-dependent bifunctional proteases are responsible for leader peptide processing of class III lanthipeptides. Proc. Natl. Acad. Sci. U S A 116 2533-2538, https://doi.org/10.1073/pnas.1815594116.

Donadio, S., Monciardini, P., and Sosio, M. (2009). Chapter 1. Approaches to discovering novel antibacterial and antifungal agents. Methods Enzymol. $458,3-28$.

Du, L., Liu, R.H., Ying, L., and Zhao, G.R. (2012). An efficient intergeneric conjugation of DNA from Escherichia coli to mycelia of the lincomycin-producer Streptomyces lincolnensis. Int. J. Mol. Sci. 13, 4797-4806, https://doi. org/10.3390/ijms13044797.

Finn, R.D., Clements, J., and Eddy, S.R. (2011). HMMER web server: interactive sequence similarity searching. Nucleic Acids Res. 39, W29-W37.

Funk, M.A., and van der Donk, W.A. (2017). Ribosomal natural products, tailored to fit. Acc. Chem. Res. 50, 1577-1586, https://doi.org/10.1021/acs. accounts.7b00175.

Geller, D., Michielin, O., and Zoete, V. (2013). Shaping the interaction landscape of bioactive molecules. Bioinformatics 29, 3073-3079, https://doi.org/ 10.1093/bioinformatics/btt540.

González-Pastor, J.E., San Millán, J.L., and Moreno, F. (1994). The smallest known gene. Nature 369, 281, https://doi.org/10.1038/369281a0.

Greule, A., Stok, J.E., De Voss, J.J., and Cryle, M.J. (2018). Unrivalled diversity: the many roles and reactions of bacterial cytochromes P450 in secondary metabolism. Nat. Prod. Rep. 35, 757-791.

Guijarro, J.I., González-Pastor, J.E., Baleux, F., San Millán, J.L., Castilla, M.A., Rico, M., Moreno, F., and Delepierre, M. (1995). Chemical structure and translation inhibition studies of the antibiotic microcin C7. J. Biol. Chem. 270, 23520-23532.

Hug, J.J., Dastbaz, J., Adam, S., Revermann, O., Koehnke, J., Krug, D., and Mueller, R. (2020). Biosynthesis of cittilins, unusual ribosomally synthesized and post-translationally modified peptides from Myxococcus xanthus. ACS Chem. Biol. 15, 2221-2231, https://doi.org/10.1021/acschembio.0c00430.
Iorio, M., Tocchetti, A., Santos Cruz, J.C., Del Gatto, G., Brunati, C., Maffioli, S.I., Sosio, M., and Donadio, S. (2018). Novel polyethers from screening Actinoallomurus spp. Antibiotics (Basel) 7, 47, https://doi.org/10.3390/antibiotics7020047.

Kase, H., Kaneko, M., and Yamada, K. (1987). K-13, a novel inhibitor of angiotensin I converting enzyme produced by Micromonospora halophytica subsp. exilisia. I. J. Antibiot. 40, 450-454.

Katoh, K., and Standley, D.M. (2013). MAFFT multiple sequence alignment software version 7: improvements in performance and usability. Mol. Biol. Evol. 30, 772-780.

Kloosterman, A.M., Shelton, K.E., van Wezel, G.P., Medema, M.H., and Mitchell, D.A. (2020). RRE-finder: a genome-mining tool for class-independent RiPP discovery. bioRxiv 14, 992123, https://doi.org/10.1101/2020.03.14. 992123.

Letunic, I., and Bork, P. (2019). Interactive Tree of Life (iTOL) v4: recent updates and new developments. Nucleic Acids Res. 2, W256-W259, https:// doi.org/10.1093/nar/gkz239.

Medema, M.H., Kottmann, R., Yilmaz, P., Cummings, M., Biggins, J.B., Blin, K., de Bruijn, I., Chooi, Y.H., Claesen, J., Coates, R.C., et al. (2015) Minimum information about a biosynthetic gene cluster. Nat. Chem. Biol. 11, 625-631.

Monciardini, P., Iorio, M., Maffioli, S.I., Sosio, M., and Donadio, S. (2014). Discovering new bioactive molecules from microbial sources. Microb. Biotechnol. 7, 209-220.

Montalbán-López, M., Scott, T.A., Ramesh, S., Rahman, I.R., van Heel, A.J., Viel, J.H., Bandarian, V., Dittmann, E., Genilloud, O., Goto, Y., et al. (2020). New developments in RiPP discovery, enzymology and engineering. Nat. Prod. Rep.. https://doi.org/10.1039/d0np00027b.

Pastor, I., Vilaseca, E., Madurga, S., Gargès, J.L., Cascante, M., and Mas, F. (2011). Effect of crowding by dextrans on the hydrolysis of N-succinyl-Lphenyl-Ala-p-nitroanilide catalyzed byr-chymotrypsin. J. Phys. Chem. B 115 (5), 1115-1121.

Price, M.N., Dehal, P.S., and Arkin, A.P. (2010). FastTree 2-approximately maximum-likelihood trees for large alignments. PLoS One 5, e9490, https:// doi.org/10.1371/journal.pone.0009490.

Reisberg, S.H., Gao, Y., Walker, A.S., Helfrich, E.J., Clardy, J., and Baran, P.S. (2020). Total synthesis reveals atypical atropisomerism in a small-molecule natural product, tryptorubin A. Science 367, 458-463.

Russell, A.H., and Truman, A.W. (2020). Genome mining strategies for ribosomally synthesised and post-translationally modified peptides. Computat. Struct. Biotechnol. J. 18, 1838-1851.

Saito, S., Atsumi, K., Zhou, T., Fukaya, K., Urabe, D., Oku, N., Karim, M.R.U., Komaki, H., and lgarashi, Y. (2020). A cyclopeptide and three oligomycin-class polyketides produced by an underexplored actinomycete of the genus Pseudosporangium. Beilstein J. Org. Chem. 16, 1100-1110.

Sano, S., Ikai, K., Katayama, K., Takesako, K., Nakamura, T., Obayashi, A., Ezure, Y., and Enomoto, H. (1986). OF4949, new inhibitors of aminopeptidase B II. Elucidation of structure. J. Antibiot. 39, 1685-1696.

Wyche, T.P., Ruzzini, A.C., Schwab, L., Currie, C.R., and Clardy, J. (2017). Tryptorubin A: a polycyclic peptide from a fungus-derived Streptomycete. J. Am. Chem. Soc. 139, 12899-12902.

Yasuzawa, T., Shirahata, K., and Sano, H. (1987). K-13, a novel inhibitor of angiotensin I converting enzyme produced by Micromonospora halophytica subsp. exilisia. II. J. Antibiot. 40, 455-458.

Yera, E.R., Cleves, A.E., and Jain, A.N. (2011). Chemical structural novelty: ontargets and off-targets. J. Med. Chem. 54, 6771-6785.

Zdouc, M.M., Iorio, M., Maffioli, S.I., Crüsemann, M., Donadio, S., and Sosio, M. (2020). Preprint bioRxiv, p. 210815, https://doi.org/10.1101/2020.07.19 210815. 


\section{STAR $\star$ METHODS}

\section{KEY RESOURCES TABLE}

\begin{tabular}{|c|c|c|}
\hline REAGENT or RESOURCE & SOURCE & IDENTIFIER \\
\hline \multicolumn{3}{|l|}{ Bacterial and virus strains } \\
\hline Escherichia coli $\mathrm{DH} 5 \alpha$ cells & Bioline & Bio-85026 \\
\hline Escherichia coli ET12567/pUZ8002 & Macneil et al., 1992 & NA \\
\hline Streptomyces coelicolor strain M1152 & Flinspach et al., 2010 & NA \\
\hline Planomonospora sp. ID82291 & Naicons collection & NA \\
\hline Planomonospora sp. ID107089 & Naicons collection & NA \\
\hline Planomonospora sp. ID114239 & Naicons collection & NA \\
\hline Planomonospora sp. ID46116 & Naicons collection & NA \\
\hline \multicolumn{3}{|l|}{ Chemicals, peptides, and recombinant proteins } \\
\hline Apramycin sulfate & Sigma-Aldrich & Cat\# A2024-5G \\
\hline Nalidixic acid & Sigma-Aldrich & Cat\# 1451000 \\
\hline Diaion HP-20 & Sigma-Aldrich & Cat\# 13,605 \\
\hline$\alpha$-chymotrypsin from bovine pancreas & Sigma-Adrich & Cat\# C4129 \\
\hline Chymostatin, microbial & Sigma-Aldrich & Cat\# C7268 \\
\hline Deuterium oxide & Sigma-Aldrich & Cat\# 151882 \\
\hline \multicolumn{3}{|l|}{ Critical commercial assays } \\
\hline Phusion DNA polymerase & NEB & Cat\# M0530S \\
\hline Taq DNA ligase & NEB & Cat\# M0208S \\
\hline T5 exonuclease & NEB & Cat\# M0663S \\
\hline $\begin{array}{l}\text { Angiotensin I Converting Enzyme (ACE) } \\
\text { Activity Assay Kit }\end{array}$ & Sigma-Aldrich & Cat\# CS0002 \\
\hline \multicolumn{3}{|l|}{ Deposited data } \\
\hline Planomonospora sp. ID82291 genome & Zdouc et al. (2020) & GenBank: JABTEX000000000 \\
\hline biarylitide-YYH gene cluster & this paper & GenBank: MW201788 \\
\hline biarylitide-YFH gene cluster & this paper & GenBank: MW201789 \\
\hline iTOL-webserver & this paper & $\begin{array}{l}\text { https://itol.embl.de/tree/ } \\
21312710611256691590408100\end{array}$ \\
\hline iTOL- Expanded clades & this paper & $\begin{array}{l}\text { https://itol.embl.de/tree/ } \\
2131271061176991591098016\end{array}$ \\
\hline \multicolumn{3}{|l|}{ Oligonucleotides } \\
\hline $\begin{array}{l}\text { pSET_fwd: } \\
\text { CCTCTCTAGAGTCGACCTGCAGC }\end{array}$ & this paper & NA \\
\hline pSET_rev: CCTTCCGTACCTCCGTTGCT & this paper & NA \\
\hline $\begin{array}{l}\text { bytAO_fwd: } \\
\text { AGCAACGGAGGTACGGAAGGAGGAGG } \\
\text { TGTGCGATGCGC }\end{array}$ & this paper & NA \\
\hline $\begin{array}{l}\text { bytAO_rev: } \\
\text { GCAGGTCGACTCTAGAGAGGCTAGCGG } \\
\text { GGGAGAAGGAC }\end{array}$ & this paper & NA \\
\hline $\begin{array}{l}\text { pSET-bytAO_fwd: } \\
\text { GCGTCGATTITTGTGATGCTCG }\end{array}$ & this paper & NA \\
\hline $\begin{array}{l}\text { pSET-bytAO_rev: } \\
\text { CAGCGAATTCGGAAAACGGC }\end{array}$ & this paper & NA \\
\hline \multicolumn{3}{|l|}{ Recombinant DNA } \\
\hline pSET152_ermE ${ }^{\star}$ & Bierman et al. (1992) & NA \\
\hline pSET-bytAO & this paper & NA \\
\hline
\end{tabular}


Please cite this article in press as: Zdouc et al., A biaryl-linked tripeptide from Planomonospora reveals a widespread class of minimal RiPP gene clusters, Cell Chemical Biology (2020), https://doi.org/10.1016/j.chembiol.2020.11.009

Cell Chemical Biology Brief Communication

\begin{tabular}{|c|c|c|}
\hline Continued & & \\
\hline REAGENT or RESOURCE & SOURCE & IDENTIFIER \\
\hline \multicolumn{3}{|l|}{ Software and algorithms } \\
\hline antiSMASH 5.0 & Blin et al. (2019) & $\begin{array}{l}\text { https://antismash. } \\
\text { secondarymetabolites.org/ }\end{array}$ \\
\hline MIBiG database & Medema et al. (2015) & https://mibig.secondarymetabolites.org/ \\
\hline MAFFT & Katoh, et al., 2013 & https://mafft.cbrc.jp/alignment/software/ \\
\hline HMMER & Finn, et al., 2011 & http://hmmer.org/ \\
\hline FastTree2 & Price, et al., 2010 & http://microbesonline.org/fasttree/ \\
\hline iTOL server & Letunic I., Bork P., 2019 & https://itol.embl.de/ \\
\hline \multicolumn{3}{|l|}{ Other } \\
\hline Mass Spectrometer & Thermo Scientific & LCQ Fleet \\
\hline $\begin{array}{l}\text { Dionex Ultimate } 3000 \text { with diode array } \\
\text { detector }\end{array}$ & Thermo Scientific & NA \\
\hline $\begin{array}{l}\text { Column Atlantis T3 C18 } 5 \mu \mathrm{m} \times 4.6 \mathrm{~mm} \\
\times 50 \mathrm{~mm}\end{array}$ & Waters & Cat\# 186003726 \\
\hline HR-LCMS & Bruker & MicroTOF-QIII \\
\hline Enspire Multimode Plate Reader & PerkinElmer & NA \\
\hline $\begin{array}{l}\text { CombiFlash Rf flash liquid chromatography } \\
\text { system }\end{array}$ & Teledyne Isco & Cat\# 68-5230-015 \\
\hline SNAP Ultra HP-Sphere C18 $25 \mu \mathrm{m} \mathrm{12g}$ & Biotage & NA \\
\hline LC-2010 AHT & Shimadzu & NA \\
\hline $\begin{array}{l}\text { Column Nucleodur C18 Pyramid } 5 \mu \mathrm{m} \times \\
250 \mathrm{~mm} \times 10 \mathrm{~mm}\end{array}$ & Macherey-Nagel & Cat\# 762272.100 \\
\hline $300 \mathrm{MHz}$ Avance NMR Spectrometer & Bruker & NA \\
\hline
\end{tabular}

\section{RESOURCE AVAILABILITY}

\section{Lead contact}

Further information and requests for reagents should be directed to and will be fulfilled by the Lead Contact, Margherita Sosio (msosio@naicons.com)

Materials availability

Materials generated in this study can be obtained with a Materials Transfer Agreement.

Data and code availability

The software used in this study are listed in the Key Resourses Table. The Planomonospora sp. ID82291 genome sequence was deposited into the GenBank with the accession number JABTEX000000000. The sequences of the biarylitide-YYH and biarylitideYFH gene clusters have been deposited in the NCBI under the accession number GenBank MW201788 and MW201789, respectively. Expanded clades and detailed annotations are accessible at: https://itol.embl.de/tree/2131271061176991591098016. Further data are available from the corresponding author upon request.

\section{EXPERIMENTAL MODEL AND SUBJECT DETAILS}

Bacterial strains and growth conditions

The bacterial strains used in this study are listed in the Key Resources Table. Planomonospora strains from frozen stocks $\left(-80^{\circ} \mathrm{C}\right)$ were propagated on $\mathrm{S} 1$ plates at $28^{\circ} \mathrm{C}$ for two to three weeks. The grown mycelium was then homogenized with a sterile pestle and used to inoculate $15 \mathrm{~mL} \mathrm{AF}$ medium ( $20 \mathrm{~g} / \mathrm{L}$ dextrose, $8 \mathrm{~g} / \mathrm{L}$ soybean meal, $2 \mathrm{~g} / \mathrm{L}$ yeast extract, $4 \mathrm{~g} / \mathrm{L} \mathrm{CaCO}, 1 \mathrm{~g} / \mathrm{L} \mathrm{NaCl}$, pH 7.2$)$ in a $50 \mathrm{~mL}$ baffled flask. After cultivation on a rotary shaker $(200 \mathrm{rpm})$ at $30^{\circ} \mathrm{C}$ for $72 \mathrm{hr}, 1.5 \mathrm{~mL}$ of the exponentially growing culture was used to inoculate each $15 \mathrm{~mL}$ of AF medium, as described (Donadio et al., 2009; Zdouc et al., 2020). After 7 days of cultivation as before, cultures were harvested and extracted by centrifugation and the supernatant, after adsorption on $200 \mu \mathrm{L}$ of HP20 resin (Diaion) for $1 \mathrm{hr}$, was eluted with $200 \mu \mathrm{L}$ of methanol (Sigma). Samples were analyzed by LC-MS as described below. For isolation and purification of 1, $1.5 \mathrm{~mL}$ of a frozen stock of strain ID82291 was used to inoculate $15 \mathrm{~mL}$ AF medium in a $50 \mathrm{~mL}$ baffled flask (Donadio et al., 2009). After $72 \mathrm{hr}, 10 \mathrm{~mL}$ were used to inoculate $100 \mathrm{~mL}$ fresh AF medium in a $500 \mathrm{~mL}$ baffled flask. After further $72 \mathrm{hr}, 100 \mathrm{~mL}$ was used to inoculate $900 \mathrm{~mL} \mathrm{AF}$ medium in a $3 \mathrm{~L}$ flask, containing $50 \mathrm{~g} \mathrm{5-mm} \mathrm{glass} \mathrm{beads.}$ 


\title{
CellPress
}

\section{Cell Chemical Biology Brief Communication}

\begin{abstract}
Cultivation in $\mathrm{D}_{2} \mathrm{O}$
$1.5 \mathrm{~mL}$ of a frozen stock culture of strain ID82291 was used to inoculate $15 \mathrm{~mL} \mathrm{AF} \mathrm{medium} \mathrm{in} \mathrm{a} 50 \mathrm{~mL}$ baffled flask. After $72 \mathrm{hr}, 1.5 \mathrm{~mL}$ were used to inoculate each $15 \mathrm{~mL} \mathrm{AF}$ medium prepared in $30 \% \mathrm{D}_{2} \mathrm{O}(\mathrm{v} / \mathrm{v})$ in $50 \mathrm{~mL}$ baffled flasks and further cultivated under identical conditions. Production of 1 was monitored by sampling $1 \mathrm{~mL}$ of broth every $24 \mathrm{hr}$. Samples were centrifuged and the supernatant, after adsorption on $200 \mu \mathrm{L}$ of HP20 resin (Diaion) for $1 \mathrm{hr}$, was eluted with $200 \mu \mathrm{L}$ of methanol (Sigma). Samples were analyzed by LCMS as described below.
\end{abstract}

\section{METHOD DETAILS}

Isolation and purification of 1

After 7 days, the culture (as described above) was first centrifuged and then filtered, and the resulting cleared broth was adsorbed on $200 \mathrm{~mL}$ HP20 resin (Diaion). The loaded resin was washed with demineralized water and eluted with aqueous $50 \% \mathrm{MeOH}(\mathrm{V} / \mathrm{V})$. The eluate was brought to dryness. The dried extract was taken up in $10 \%$ acetonitrile/90\% water and pre-fractionated on a Biotage SNAP Ultra HP-Sphere C18 $25 \mu \mathrm{m} \mathrm{12g} \mathrm{with} \mathrm{a} \mathrm{CombiFlash} \mathrm{system} \mathrm{(Teledyne} \mathrm{ISCO,} \mathrm{Nebraska,} \mathrm{USA).} \mathrm{All} \mathrm{chromatographic} \mathrm{steps}$ were performed using purified water (MilliQ, Merck). Phases A and B were water and acetonitrile (Sigma-Aldrich), respectively. A gradient with steps of 5, 5, 30, 100 and $100 \%$ phase $B$ at 0, 5, 20, 25 and 30 min, respectively, was applied. After screening fractions by LC-MS, 1-containing fractions were dried, taken up in $0.05 \%$ trifluoroacetic acid (TFA) in $10 \%$ MeCN/90\% water and processed further by semi-preparative HPLC on a Shimadzu LC-2010 CHT instrument, equipped with a 250/10 Nucleodur C18 Pyramid 5M column (Macherey-Nagel) and a C18 pre-column. An isocratic method with 10\% phase B (A: 0.05\% TFA in water, $\mathrm{pH} 2.5$; $\mathrm{B}$ : MeCN) at $3 \mathrm{~mL} / \mathrm{min}$, column temperature $40^{\circ} \mathrm{C}$ was used for purification. Due to co-eluting contaminants, the semi-purified substance was purified for a second time under the same conditions, except for the change of phase A to water. Drying of the eluent yielded $5 \mathrm{mg}$ of a white solid.

\section{Analytical procedures}

High-resolution MS spectra acquisition was performed on a micrOTOF-Q III (Bruker) instrument equipped with an electrospray interface (ESI) coupled with a Dionex UltiMate 3000 (Thermo Scientific), using an Atlantis T3 C18 $5 \mu \mathrm{m} \times 4.6 \mathrm{~mm} \times 50 \mathrm{~mm}$ column (Zdouc et al., 2020). LC-MS measurements for monitoring metabolite production were performed on a Dionex UltiMate 3000 (Thermo Scientific) coupled with an LCQ Fleet (Thermo Scientific) mass spectrometer equipped with an electrospray interface (ESI) on an Atlantis T3 C18 $5 \mu \mathrm{m} \times 4.6 \mathrm{~mm} \times 50 \mathrm{~mm}$ column, as reported elsewhere (lorio et al., 2018). LC-MS measurements for analysis of 1 after acid hydrolysis were performed on the same instrument, applying a hydrophilic gradient with $0,0,25,95,95,0$ and $0 \%$ phase $B$ (MeCN) at $0,1,7,8,12,12.5$ and $14 \mathrm{~min}$, respectively, with the $\mathrm{m} / \mathrm{z}$ range set to $110-1000 \mathrm{Da}$. Phase A was $0.05 \%$ TFA in water. UV-VIS signals $(190-600 \mathrm{~nm})$ were acquired using the built-in diode array detector of the Dionex UltiMate 3000 (Thermo Scientific). Mono- and bidimensional NMR spectra were measured in DMSO- $d 6$ or $\mathrm{CD}_{3} \mathrm{OD}$ at $298 \mathrm{~K}$ using a Bruker Avance $300 \mathrm{MHz}$ spectrometer.

\section{Hydrolysis of 1}

A small amount of purified compound $\mathbf{1}(<1 \mathrm{mg}$ ) was dissolved in $250 \mu \mathrm{L} 0.1 \mathrm{M}$ sodium borate buffer (pH 9), followed by addition of $250 \mu \mathrm{L}$ fresh $6 \mathrm{~N} \mathrm{HCl}$ (Sigma-Aldrich). The solution was heated to $120^{\circ} \mathrm{C}$ and sampled at 24,48 and $96 \mathrm{hr}$. The samples were analyzed by LC-MS analysis, using the hydrophilic gradient as described above.

\section{Biarylitide YYH (1)}

white crystalline powder; $[\alpha]_{D}=-165\left(\mathrm{c} 0.11, \mathrm{H}_{2} \mathrm{O} \mathrm{pH} 10,25^{\circ} \mathrm{C}\right) ;{ }^{1} \mathrm{H}$ and ${ }^{13} \mathrm{C}$ NMR data in Supporting Information (Figures S2 and S3); HR-ESI-MS m/z 522.198 [M + H] $]^{+}$(calcd for $\mathrm{C}_{26} \mathrm{H}_{27} \mathrm{~N}_{5} \mathrm{O}_{7}, 522.1988$ ); ESI-MS/MS ([M + H] $]^{+}$m/z (\%) 269.139 (100), 359.134 (84), 315.143 (69), 494.201 (66), 289.128 (61), 285.133 (56), 331.140 (54), 243.122 (51), 313.129 (37), 448.198 (27), 476.191 (17), 228.111 (10), 406.181 (9), 214.094 (9), 199.081 (7).

\section{Cloning and heterologous Expression of bytAO}

A DNA stretch containing bytAO (1303 bp) was cloned into pSET152_ermE* via Gibson assembly. Linear DNA fragments were generated by PCR using primers designed to include 20 bp overlap sequences (pSET_fwd: CCTCTCTAGAGTCGACCTGCAGC, pSET_rev: CCTTCCGTACCTCCGTTGCT, bytAO_fwd: AGCAACGGAGGTACGGAAGGAGGAGGTGTGCGATGCGC and bytAO_rev: GCAGGTCGACTCTAGAGAGGCTAGCGGGGGAGAAGGAC). Purified linear vector and insert were mixed (ratio 1:3) with $15 \mu \mathrm{L}$ of Gibson assembly master mixture (1X ISO buffer, $10 \mathrm{U} / \mathrm{L}$ T5 exonuclease, $2 \mathrm{U} / \mathrm{L}$ Phusion polymerase, and $40 \mathrm{U} / \mu \mathrm{L}$ Taq ligase) and incubated at $50^{\circ} \mathrm{C}$ for $1 \mathrm{hr}$. Then, $5 \mu \mathrm{L}$ of reaction mix were used to transform chemically competent $E$. coli DH5 $\alpha$ cells, which were plated onto LB agar supplemented with $50 \mu \mathrm{g} / \mathrm{mL}$ apramycin. Positive clones containing the plasmid pSET-bytAO were selected by colony PCR (primers: pSETbytAO_fwd: GCGTCGATTITGTGATGCTCG and pSET-bytAO_rev: CAGCGAATTCGGAAAACGGC) and confirmed using restriction digest and sequencing. pSET-bytAO was conjugated into S. coelicolor M1152 using a standard intergeneric conjugation protocol (Du et al., 2012) with the methylation-deficient E. coli ET12567/pUZ8002/pSET-bytAO as the donor. Cells were spread onto MS agar (20 g/L soya flour, $20 \mathrm{~g} / \mathrm{L}$ mannitol, $20 \mathrm{~g} / \mathrm{L}$ agar, $50 \mathrm{mM} \mathrm{MgCl}$ ) and incubated at $30^{\circ} \mathrm{C}$. After $20 \mathrm{hr}, 0.5 \mathrm{mg}$ nalidixic acid and $1 \mathrm{mg}$ apramycin were overlaid on the plates and incubation continued until ex-conjugants showed up. Single colonies were spread on fresh plates containing $25 \mathrm{mg} / \mathrm{L}$ nalidixic acid and $50 \mathrm{mg} / \mathrm{L}$ apramycin to confirm the ex-conjugants. Colonies containing byt $A O$ 
were validated by colony PCR and cultivated in TSB broth for 12 days at $30^{\circ} \mathrm{C}$. Cultures were extracted as described above and analyzed by HR-LCMS, as described above.

\section{Protease activity assay}

Angiotensin I Converting Enzyme (ACE) inhibition was measured using the Sigma-Aldrich Angiotensin I Converting Enzyme (ACE) Activity Fluorescence Assay Kit (CS0002), following instruction. Briefly, a serial dilution of compound 1, starting at $100 \mu \mathrm{M}$, was tested for inhibitory activity against ACE. As a positive control, teptrotide (Sigma-Aldrich A0773) at $100 \mu \mathrm{M}$ was used. Reactions were incubated at $37^{\circ} \mathrm{C}$ and measured every 5 min, using a PerkinElmer Enspire Multimode Plate Reader. All reactions were performed in duplicates and the mean calculated. Chymotrypsin inhibition was measured by a fluorescent-based assay (Pastor et al., 2011). Briefly, a serial dilution of compound 1, starting at $100 \mu \mathrm{M}$, was tested for inhibitory activity against $\alpha$-chymotrypsin from bovine pancreas (Sigma-Adrich C4129). As a positive control, chymostatin from microbial origin (Sigma-Aldrich C7268) at $40 \mu \mathrm{g} / \mathrm{mL}$ was used. Reactions were incubated at $25^{\circ} \mathrm{C}$ and measured every $5 \mathrm{~min}$, using a PerkinElmer Enspire Multimode Plate Reader. All reactions were performed in duplicates and the mean calculated.

\section{Phylogenetic analyses}

A set of complete genomes from the antiSMASH database were used along with top blast results to PLM4_2056 from Planomonospora sp. ID82291 (NCBI reference JABTEX000000000). Cytochrome P450 enzymes were identified via a hidden Markov model search (Finn et al., 2011) using the Pfam (PF00067) P450 model and trusted bit-score cutoffs. After ensuring model and query alignment coverage over $70 \%$, similar sequences (>95\% amino acid ID) were clustered via an all vs. all blast and one representative was used for the final dataset, which yielded over 3,300 sequences. Any hit to the motif search analysis (see below) were also included in this dataset. All sequences were aligned via MAFFT (Katoh et al., 2013) using the local iterative (-linsi) option followed by alignment trimming using the trimal application with automated option to improve maximum-likelihood tree inference. Due to the large number of sequences FastTree (Price et al., 2010) was used to infer the final phylogenetic gene tree.

\section{Peptide motif search}

A custom python script (https://git.wur.nl/snippets/65) was used to examine 6-frame translations of corresponding genomic regions $+/-500 b p$ of identified cytochrome P450 enzymes. This reported any motif matching MxYx[Y/H $]^{*}$, where " $x$ " is any amino acid and * is a stop codon. Reported positions were then cross-referenced to highlight motifs occurring up/downstream of the P450 CDS. An additional screen of these results for the Shine-Dalgarno sequence "AGGAGG" was preformed upstream of all motif positions to annotate RBS sites. Identified peptides were mapped onto the generated, P450-based phylogenetic tree and visualized using the iTOL-webserver (Letunic and Bork, 2019; iTOL Webserver. https://itol.embl.de/, Accessed: 06/11/2019).

\section{QUANTIFICATION AND STATISTICAL ANALYSIS}

PerkinElmer Enspire Multimode Plate Reader was used for the Angiotensin I Converting Enzyme (ACE) Activity Fluorescence Assay and for the Chymotrypsin inhibition Assay, in METHOD DETAILS (Protease Activity Assay): experiments were conducted in duplicates and the mean calculated using Microsoft Excel. 